\title{
Caribbean Itch: Eight Cases And One Who Didn't (Exercise Blue Calypso Diamond)
}

\author{
Maj NJ Jefferies \\ $M B, B S, F R C A$, RAMC \\ Consultant Anaesthetist, Head of Department
}

BMH Rinteln, BFPO 31.

\author{
Sgt N Rushby \\ RAMC
}

35 Engr Regt, BFPO 31.

SUMMARY: Stinging plankton are a potential source of irritation and morbidity for those pursuing aquatic sports in areas where they are numerous. The problem is seasonal, and likely to be familiar to local people. We describe an incident in which a number of military personnel were affected by the stings of the thimble jelly fish (Linuche $\stackrel{?}{-}$ unguiculata), with a brief review of the biology of the problem and some recommendations for prevention.

\section{Case Report}

Exercise Blue Calypso Diamond, in May 1996, was planned as two weeks of scuba-diving training and qualification around the Belize barrier reef. Nine members of the Hameln Garrison Sub-Aqua Club took part, all males and aged from 27 to 44 years, and the exercise was based at the British Army Adventure Training Centre on St George's Cay. All participants were in good health and taking no medication apart from the recommended antimalarial prophylaxis.

During diving, eight of the nine wore wetsuits made of neoprene fabric $6 \mathrm{~mm}$ thick which covered the torso and upper thighs. Boots of similar material were worn by all divers as well as gloves when appropriate. The remaining member of the party was unable to find a wetsuit to fit and so went in a t-shirt and athletic training trousers.

While in the water we were aware of occasional brief sensations of contact, without any visible cause, followed by mild irritation. Over the following few days eight of us developed an itchy, erythematous papular rash on the exposed areas of the body with a concentration around the collar line. The man wearing the $t$-shirt and training trousers was, however, affected over much of his body. The itching prevented him from sleeping and he was forced to abandon his diving programme by mild constitutional symptoms. Physical examination revealed nothing apart from the rash, and he was apyrexial. He received symptomatic treatment with antihistamines (chlorpheniramine and astemizole) with some relief. Nobody suffered scarring or lasting ill-effects and the rash had faded in all cases within a few days of leaving the area.

Local enquiries revealed that this problem was wellknown and called "pika-pika", and thought to be due to plankton, but without any specific organism being implicated. The member of our party who emerged unscathed had taken the advice of a local boatman and had applied sun-cream liberally over his exposed areas (the original recommendation had been for Vaseline, but suncream was an available alternative).

During our travels in the area we encountered to Operation Raleigh team who were living on Half Mosign Cay and heard that several of them had also been affectead. 음 It may be significant that those who were worst affect 9 음 seemed to be the fair-skinned.

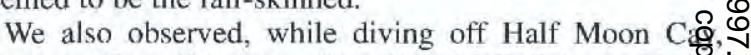
numbers of small (approx. $2 \mathrm{~cm}$ diam.) jellyfish notable their dark brown internal colouring. These were seen bo as isolated specimens and as swarms which probabty contained several thousand individuals drifting together at the surface clustered as an inverted cone. We identified $\frac{\text { O }}{0}$ them retrospectively as the thimble jellyfish Linuche $\stackrel{\Omega}{\rightarrow}$ unguiculata which inhabits the Caribbean and breeds $\overline{\bar{B}}$ throughout the summer with a peak in May.

This, or its tiny larva, is known as a cause of problems for swimmers (1) and was probably responsible for ours.

\section{Discussion}

The hazards of scuba diving include those implicit in entering the aquatic environment and depending for survival on the performance of specialised equipment; and those related to the life forms that have evolved biological $\overrightarrow{0}$ mechanisms to feed or to defend themselves in an 3 . unforgiving world of predators and prey. The teeth of 8 sharks and the venoms of water snakes are at least attached to highly visible creatures: some unpleasant surprises areo much less visible either because of camouflage and concealment (e.g. stone-fish and their poisonous spines) or because of small size. Stinging plankton, which may be the larvae of larger animals, are in this last category.

Jellyfish are aquatic invertebrates of the phylum $\tilde{O}$ Coelenterata (Gk. "hollow gut"), which includes the sea N anemones, hydra and corals. They are typically bell- 
shaped and range widely in size, the largest being up to $2 \mathrm{~m}$ in diameter. They are characterised by variably numbered tentacles which hang from the margin of the bell, with a central manubrium which hangs down and supports the mouth. They have sense organs which respond to gravity and to light, and move by rhythmic contractions of the margin which jet-propel them through the water. Salt- and freshwater species exist, with fossil examples among the earliest multicellular life-forms.

The free-swimming "medusa" is the sexual stage in the animal's life cycle. Eggs and sperm are produced which unite and develop into larvae, which drift almost invisibly as part of the plankton population. Some species pass through a sedentary polyp stage, usually very small, which buds asexually to produce new medusae: in others the larva matures directly into the sexual form.

All the coelenterates are carnivorous, and their tentacles and mouthparts bear stinging cells, known as cnidocytes, which are used to trap prey and give rise to the phylum's alternative name of Cnidaria, or "nettle". The cnidocytes contain a coiled, hollow nematocyst which everts in response to touch, or to a chemical or electrical stimulus. This pierces the victim's flesh and releases poison. These structures are present in both adults and larvae.

The stings of four genera: Chironex fleckeri (the Australian box jellyfish); Chiropsolmus quadrigatus and C. quadrumanus (SE Asia); Stomolouphus nomurai (SE China); and Physalia physalis (the Portuguese man-o-war, which lives in the western Atlantic and is actually a floating colony made up of a number of specialised individuals) are sufficiently powerful to be lethal to humans, but nothing so dangerous regularly inhabits the Caribbean.

The venoms are often proteins, and their actions are varied. Our assailant seemed to cause mainly local histamine release, but there are other substances with other actions including $\mathrm{Na} / \mathrm{K}$ ATP-ase (2) and potassium channel (3) inhibition. Many have been synthesised, and because of their selectivity at receptor sites some have rôles as investigational molecular probes (3). A single species may produce more than one toxin: the nematocyst of the boxjellyfish has been reported to contain two myotoxins (MW 6000000 and 150000), and a haemolysin (MW 70000) (4).

Treatment of severe envenomation involves resuscitation, careful removal of adherent tentacles (which can still bear active nematocysts!), vinegar dousing to inhibit nematocyst discharge, and the use of specific antivenom (5). Current antivenoms are raised in sheep, with the attendant hazard of hypersensitivity to ovine serum, but there has been some progress in production of monoclonal antibodies to constituents of the box jellyfish sting (6).

\section{Conclusion}

Most of our party were merely made uncomfortable, but the exercise was disrupted for one of us and it is appropriate to pass on our observations in the hope that others may not be so inconvenienced.

1) The creature responsible for these stings does not seem able to penetrate neoprene wet suit material, although thinner fabrics are not protective and there does seem to be a "streaming" effect (hence the concentration of stings around the collar). Appropriate clothing should be worn when diving in the area, and should be fully fastened.

2) The application of sun-cream (or Vaseline) before entering the water also seems to be useful protection.

3) The problem is seasonal, with May being the peak breeding season for these animals and therefore the most hazardous time.

4) It is often worth while seeking local advice about local problems.

\section{REFERENCES}

1. Macsween R, Williams H. Seabathers, eruption. $B$ Med J 1996; 312: 957-8.

2. Terao KK, ito E, Yasumoto $T$, Light and electronmicroscopic observation of experimentah palytoxin poisoning in mice. Bull Soc Pathol Exoß Filiales 1992; 85: 494-6.

3. Pennington MW, Byrnes ME, Zaydenberg I, et a Chemical synthesis and characterization of ShK toxin: a potent $\mathrm{K}+$ channel inhibitor from a sea anemone. Int $J$ Pept Protein Res 1995; 46: 354-8.

4. Endean R, MonKs SA, Cameron aM. Toxins from the boxjellyfish Chironex fleckeri. Toxicon 1993; 31; 397-410.

5. Warrell DA. Venomous bites and stings. Med Int 1992;107: 4529-33.

6. Collins SP, Comis A, Marshall M, Hartwick RF, HowDEN ME. Monoclonal antibodies neutralizing the haemolytic activities of box jellyfish (C. fleckeri) tentacle extracts. Comp Biochem Physiol 1993; 106B: 67-70. 